\title{
ADAPTIVE ANTENNAS AT THE MOBILE AND BASE STATIONS IN AN OFDM/TDMA SYSTEMS
}

\author{
K-K Wong, R. S-K Cheng, K. Ben Letaief, and R. D. Murch \\ Department of Electrical \& Electronic Engineering \\ The Hong Kong University of Science \& Technology \\ Clear Water Bay, Kowloon, Hong Kong \\ Email: eewkkit@ee.ust.hk
}

\begin{abstract}
In recent years, several smart antenna systems have been proposed, and demonstrated at the base station of wireless communication systems, and these have shown that significant system performance improvement is possible. In this paper, we consider the use of adaptive antennas at the base and mobile stations in combination with orthogonal frequency division multiplexing in both the down and uplinks. This system allows multiple user access in frequency subcarriers as well as space. Thus, it is also known as spatialsubcarrier multiple access. The advantages of the proposed system includes reductions in average error probability and increases in capacity. To demonstrate the potential of our proposed system, analytical along with Monte Carlo simulation results are provided.
\end{abstract}

\section{INTRODUCTION}

The next-generation wireless communication systems will need to be able to support a high level of user traffic along with a wide range of high-quality services which not only include high-quality voice but also data, facsimile, and video. Providing these high quality services over wireless channels with a limited spectrum implies that an increase in the capacity of current wireless systems will need to be achieved [1], [2].

Currently, one possible approach to increase system capacity is through the use of smart or adaptive antennas [3], [4]. In this paper, we investigate the performance of adaptive antennas at both the base station (BS) and mobile station (MS) in the up and downlinks with orthogonal frequency division multiplexing (OFDM) [5]. Smart antennas at the BS and MS in conjunction with OFDM operate jointly to maximize the signal-to-interference plus noise ratio (SINR) without an equalizer, therefore cancelling cochannel interference (CCI) while intersymbol interference (ISI) is prohibited through the use of OFDM. We will refer to this system with a smart base and smart mobile (SBM) antennas and OFDM as SBM/OFDM.

The bit error rate (BER) performance of our proposed system is found for both flat fading and fre- quency selective fading channels and compared to a conventional single carrier system with smart antennas. Closed form solutions are provided for the antenna weights in flat fading channels. In this paper, adaptive modulation or dynamic power distribution among subcarriers of the OFDM system is not considered to simplify the analysis.

This paper is organized as follows. In Section II, the system model of SBM/OFDM is introduced. Section III provides analytical expressions for optimal antenna weight vectors for SBM in frequency selective and non-selective fading environments with CCI. In Section IV, a technique that reduces the computing complexity of SBM/OFDM is proposed. Comparative simulation results are presented in Section V. Finally, we have some concluding remarks in Section VI.

\section{SYSTEM MODEL OF SBM/OFDM}

The modem configuration of SBM/OFDM is shown in Figure 1. A serial-to-parallel buffer segments the $N_{f}$ information bit sequence into $N_{c}$ frames. Each parallel output stream can, in general, contain a different number of bits $m_{c}$, be independently encoded/interleaved, and be regarded as a symbol and mapped into one of the $2^{m_{c}}$ constellation points. Here, however, we set $m_{c}=2$ for all $c$ to be succinct. Beamforming with $K$ antenna weights (see next paragraph for details) is then performed, so that $K$ parallel sets of $N_{c}$ streams are formed. These bit streams are then modulated with different subcarriers by passing through inverse fast Fourier transform (IFFT) processors. A cyclic prefix is also included (the guard period is set to the excess delay of the radio channel) and the bit streams are then converted from parallel-to-serial for final transmission.

The detailed configuration of the beamforming module is shown in Figure 2(a) where $K$ antennas are located at the BS and the cth subcarrier signal transmitted by the $k$ th antenna in the $n$th symbol interval is multiplied by a controllable complex weight, $a_{k}^{c}(n)$. For convenience, we write the antenna weights at the BS as a vector, so that

$$
\mathbf{a}_{c}(n)=\left[\begin{array}{llll}
a_{1}^{c}(n) & a_{2}^{c}(n) & \cdots & a_{K}^{c}(n)
\end{array}\right]^{T}
$$

where the superscript $T$ denotes the transpose opera- 
tion.

In total, this implies there would be $N_{c} \times K$ weights with the corresponding suggestion that a heavy computation load would be required as compared to a single carrier system. However, several possibilities exist for reducing this load. For example, by exploiting the correlation between adjacent subcarrier channels, it is possible to use the same weight for a number of subcarriers, therefore, reducing the overall computation (will be discussed in Section IV).

At the receiver side, the cyclic prefix of each received signal is removed, then the subcarrier signals pass to the fast fourier transform (FFT) processors. The output symbols from the different antennas are weighted and combined to maximize SINR. The transmitted data are estimated from the combined symbols and finally, the detected bits are converted back into serial form.

The detailed configuration of the weighted combining is shown in Figure 2(b) where there are $L$ antennas and the $c$ th subcarrier signal received by the $\ell$ th antenna in the $n$th symbol interval is multiplied by a controllable complex weight, $b_{\ell}^{c}(n)$. The weighted signals from all $L$ antennas are summed to form a scalar output $s_{o, c}(n)$ and we write the receiver weights in vector notation as

$$
\mathbf{b}_{c}(n)=\left[\begin{array}{lllll}
b_{1}^{c}(n) & b_{2}^{c}(n) & \cdots & b_{L}^{c}(n)
\end{array}\right]^{T} .
$$

The wireless communication channel is here characterized by a multipath fading model. For a particular channel, the multipath model is represented by its channel impulse response using a $N$-ray model defined as

$$
c_{k, \ell}(t)=\sum_{m=0}^{N-1} \beta_{k, \ell}^{m} \delta\left(t-\tau_{k, \ell}^{m}\right)
$$

where the subscripts $k, \ell$ refer to the channel between the $k$ th and $\ell$ th antenna at the BS and MS, respectively and $N$ is the total number of paths [6]. Likewise, $\beta_{k, \ell}^{m}$ and $\tau_{k, \ell}^{m}$ are, respectively, the complex gain and time delay for the $m$ th path of the diversity channel.

To determine $\beta_{k, \ell}^{m}$, we use a statistical approach, and assume that paths with different delays are uncorrelated (i.e., uncorrelated scattering). In addition, we assume that the paths are uncorrelated for each antenna branch so as to provide perfect spatial diversity. We model $\beta_{k, \ell}^{m}$ statistically by zero mean, complex Gaussian random variables, with their power following the exponential delay profile given by

$$
\mathrm{E}\left[\left|c_{k, \ell}(t)\right|^{2}\right]=\left\{\begin{array}{cc}
\frac{1}{D} \exp \left(-\frac{t}{D}\right) & \text { for } t \geq 0 \\
0 & \text { elsewhere }
\end{array}\right.
$$

in which $D \triangleq \tau / T$ where $\tau$ and $T$ are the $r m s$ delay spread and symbol period, respectively. Moreover, we assume a constant increment delay, so $\tau_{k, \ell}^{m}=\frac{5 D}{N-1} m$.
We let $\mathbf{H}(m)$ represent the channel matrix for the $m$ th symbol, so that

$$
\mathbf{H}(m)=\left[\begin{array}{cccc}
h_{1,1}(m T) & h_{1,2}(m T) & \cdots & h_{1, L}(m T) \\
h_{2,1}(m T) & h_{2,2}(m T) & \cdots & h_{2, L}(m T) \\
\vdots & & \ddots & \vdots \\
h_{K, 1}(m T) & \cdots & & h_{K, L}(m T)
\end{array}\right]
$$

where $h_{k, \ell}(t)$ is equal to $\left(g \otimes c_{k, \ell}\right)(t)$ in which $c_{k, \ell}(t)$ is defined in $(3), g(t)$ is a raised cosine rolloff pulse shaping function, and $\otimes$ denotes the convolution operator between two continuous time signals. Usually, $h_{k, \ell}(t)$ is known as the composite channel impulse response which includes the channel as well as the pulse shaping function.

Interference is also considered and $P$ interferers are assumed. For now, we consider the downlink only. However, the uplink will be similar. The interfering channel to the $\ell$ th antenna of the desired mobile also has multipath and is defined similar to (3). Hence, the channel vector from the $p$ th interferer is

$$
\tilde{\mathbf{h}}_{p}(m)=\left[\begin{array}{llll}
\tilde{h}_{p}^{1}(m T) & \tilde{h}_{p}^{2}(m T) & \cdots & \tilde{h}_{p}^{L}(m T)
\end{array}\right]^{T}
$$

where $\tilde{h}_{p}^{\ell}(m T)$ is the composite channel impulse response representing the $p$ th interference to the $\ell$ th antenna of the desired MS link at time $t=m T$.

In the context of a QPSK signaling scheme, the $c$ th subcarrier transmitted baseband signal is

$$
s_{c}(t)=\sum_{n} z_{c}(n) g(t-n T)
$$

where $\left\{z_{c}(n)\right\}$ is the complex symbol sequence having the values of $\pm 1 \pm j$ at the $c$ th sub-band and $g(t)$ is the pulse shaping function described earlier.

In this paper, a TDMA based transmission system is assumed. In particular, data are transmitted in packets over the radio channel and these packets may include a training sequence for equalization and/or synchronization purposes along with information data. We assume the channel is quasi-stationary so that the channel is time-invariant over a packet.

\section{OPTIMAL SBM/OFDM SOLUTION}

Using the system model described in Section II, our objective is to find the optimum BS and MS weight vectors $\left(\mathbf{a}_{c}, \mathbf{b}_{c}\right)$ which maximize the overall SINR with the constraint of a fixed transmitter power $P_{T}$. In the downlink, this is expressed as

$$
\left(\mathbf{a}_{c}, \mathbf{b}_{c}\right)_{\text {opt }}=\arg \underset{\mathbf{a}_{c},\left\|\mathbf{b}_{c}\right\|^{2}=P_{T}}{\max }\{\mathrm{SINR}\}
$$

From this solution, we can determine the overall BER performance of SBM and compare it to a conventional adaptive antenna system at the BS only. Since the analysis will be the same for each subcarrier, it 
is best to think in terms of a single carrier and then later, combine together to form a multicarrier system. In our analysis, we assume that the desired channel matrix $\mathbf{H}(m)$ and the channel vector $\tilde{\mathbf{h}}_{p}(m)$ for all $P$ interferers are known. In practice, however, we will use pilot tones/symbols to estimate the channel [7].

\section{III-A. Single Subcarrier (Flat Fading)}

In a flat fading radio environment, such as a subcarrier of OFDM in a frequency selective radio environment, ISI is negligible. Hence, only the magnitude and phase of the channel at the subcarrier frequency of interest is important. For this reason, we write $\underline{\mathbf{H}}_{c}$ and $\underline{\tilde{\mathbf{h}}}_{p, c}$ as the magnitude and phase of the inverse Fourier transform of $\{\mathbf{H}(m)\}$ and $\left\{\tilde{\mathbf{h}}_{p}(m)\right\}$ at the $c$ th subcarrier. Using this notation, the received $c$ th subcarrier signal can be written as

$$
\begin{aligned}
s_{o, c}(n) & =z_{c}(n) \mathbf{b}_{c}^{T}(n) \underline{\mathbf{H}}_{c}^{T} \mathbf{a}_{c}(n) \\
& +\sum_{p=1}^{P} \tilde{z}_{p, c}(n) \mathbf{b}_{c}^{T}(n) \underline{\tilde{\mathbf{h}}}_{p, c}+\mathbf{b}_{c}^{T}(n) \mathbf{x}_{n, c}(n \gamma 9)
\end{aligned}
$$

where $\mathbf{x}_{n, c}(n)$ is complex AWGN noise.

Given $\underline{\mathbf{H}}_{c}$ and $\underline{\tilde{\mathbf{h}}}_{p, c}$, the average SINR for the $c$ th subcarrier signal can be written as

$$
\operatorname{SINR}_{c}=\frac{\sigma_{z_{c}}^{2} \mathbf{b}_{c}^{H}(n)\left(\underline{\mathbf{H}}_{c}^{T} \mathbf{a}_{c}(n)\right)^{*}\left(\underline{\mathbf{H}}_{c}^{T} \mathbf{a}_{c}(n)\right)^{T} \mathbf{b}_{c}(n)}{\mathbf{b}_{c}^{H}(n)\left[\sum_{p=1}^{P} \sigma_{\tilde{z}_{p, c}}^{2} \underline{\tilde{\mathbf{h}}}_{p, c}^{*} \underline{\tilde{\mathbf{h}}}_{p, c}^{T}+\sigma_{n}^{2} \mathbf{I}\right] \mathbf{b}_{c}(n)}
$$

where $*$ and $H$ denote the complex conjugate and complex hermitian operators, respectively, and $\mathbf{I}$ is an identity matrix. In the above equation, $\sigma_{z_{c}}^{2} \triangleq$ $\mathrm{E}\left[\left|z_{c}(n)\right|^{2}\right]$ and $\sigma_{\tilde{z}_{p, c}}^{2} \triangleq \mathrm{E}\left[\left|\tilde{z}_{p, c}(n)\right|^{2}\right]$ are defined as the digital symbol power of the desired user and the $p$ th interfering users at the cth subcarrier frequency, respectively. Also, AWGN with variance $\sigma_{n}^{2}$ is assumed.

By utilizing a standard result in [8], the weight vector, $\mathbf{b}_{c}(n)$, which maximizes the output SINR is

$$
\left.\mathbf{b}_{c}(n)\right|_{\mathrm{SINR}_{\max }}=\mu \boldsymbol{\Phi}_{u, c}^{-1}\left(\underline{\mathbf{H}}_{c}^{T} \mathbf{a}_{c}(n)\right)^{*}
$$

where $\boldsymbol{\Phi}_{u, c} \triangleq \sum_{p=1}^{P} \sigma_{\tilde{z}_{p, c}}^{2} \underline{\tilde{\mathbf{h}}}_{p, c}^{*} \underline{\tilde{\mathbf{h}}}_{p, c}^{T}+\sigma_{n}^{2} \mathbf{I}$ is defined as the correlation matrix of the undesired signal for the cth subcarrier, and $\mu$ is an arbitrary real constant.

In other words, we can find the optimum weights $\mathbf{b}_{c}(n)$ given that $\mathbf{a}_{c}(n)$ is known. The difficulty, however, is how to determine the optimum $\mathbf{a}_{c}(n)$ and the remainder of this section discusses this.

In general, $\boldsymbol{\Phi}_{u, c}$ is a hermitian and invertible matrix. Thus, $\left(\boldsymbol{\Phi}_{u, c}^{-1}\right)^{*}$ is also a hermitian matrix as is $\mathbf{A}_{c} \triangleq \underline{\mathbf{H}}_{c}^{*}\left(\boldsymbol{\Phi}_{u, c}^{-1}\right)^{*} \underline{\mathbf{H}}_{c}^{T}$. As a result, it follows that $\mathbf{A}_{c}$ can be decomposed into

$$
\mathbf{A}_{c}=\Psi_{c} \Lambda_{c} \Psi_{c}^{H}
$$

where $\boldsymbol{\Psi}_{c}$ is an unitary matrix whose columns are the eigenvectors of $\mathbf{A}_{c}$ and $\boldsymbol{\Lambda}_{c}$ is a diagonal matrix which contains the corresponding eigenvalues. Consequently,

$$
\begin{aligned}
\operatorname{SINR}_{c} & =\sigma_{z_{c}}^{2}\left(\Psi_{c}^{H} \mathbf{a}_{c}(n)\right)^{H} \boldsymbol{\Lambda}_{c}\left(\Psi_{c}^{H} \mathbf{a}_{c}(n)\right) \\
& =\sigma_{z_{c}}^{2} \mathbf{a}_{c}(n)^{H} \boldsymbol{\Lambda}_{c} \dot{\mathbf{a}}_{c}(n) \\
& =\sigma_{z_{c}}^{2}\left(\lambda_{1}\left|\dot{a}_{1}^{c}(n)\right|^{2}+\cdots+\lambda_{K}\left|\dot{a}_{K}^{c}(n)\right|^{2}(13)\right.
\end{aligned}
$$

where $\mathbf{a}_{c}(n)$ is equal to $\boldsymbol{\Psi}_{c}^{T} \mathbf{a}_{c}(n)$ and $\left\{\lambda_{k}\right\}_{k=1}^{K}$ are the eigenvalues of $\mathbf{A}_{c}$. Our constant transmitted power constraint, $\left\|\mathbf{a}_{c}(n)\right\|^{2}=P_{T}$ then becomes $\left\|\mathbf{a}_{c}(n)\right\|^{2}=$ $P_{T}$. Maximizing the SINR (13) can then be performed by using standard theory [9] so that the optimum transmission weight vector is

$$
\left.\mathbf{a}_{c}(n)\right|_{\mathrm{SINR}_{\text {max }}}=\sqrt{P_{T}} \mathbf{v}\left(\mathbf{A}_{c}\right)
$$

where $\mathbf{v}\left(\mathbf{A}_{c}\right)$ is the eigenvector of the matrix $\mathbf{A}_{c}$ which corresponds to the largest eigenvalue. Throughout, we will refer to $\mathbf{v}\left(\mathbf{A}_{c}\right)$ as the principal eigenvector of the matrix $\mathbf{A}_{c}$. The corresponding maximum SINR is

$$
\left.\mathrm{SINR}_{c}\right|_{\text {max }}=\sigma_{z_{c}}^{2} P_{T} \cdot \max _{k}\left\{\lambda_{k}\right\}
$$

Hence, the maximum SINR we can achieve with the constraint $\left\|\mathbf{a}_{\mathbf{c}}^{\prime}(n)\right\|^{2}=P_{T}$ is given by (15) and is achieved by using the weight vectors given by (11) and (14).

\section{III-B. Single Carrier (Frequency Selective Fading)}

In frequency selective fading channels such as in a single carrier system, the received signal includes multiple versions of the transmitted waveform which are attenuated and delayed in time. In this section, we specifically consider SBM for a single-carrier system in a multipath fading channel for comparison with OFDM. In general, the optimization problem (8) cannot be expected to have a closed form solution for the weight vectors as in the case of flat fading. Fortunately, approximate solutions can be provided. In particular, assuming that the transmission weights $\mathbf{a}_{c}(n)$ are fixed, then the reception weight vector which maximizes the SINR is

$$
\left.\mathbf{b}_{c}(n)\right|_{\operatorname{SINR}_{\max }, \mathbf{a}_{c}(n)}=\mu_{1} \boldsymbol{\Phi}_{c, 1}^{-1}\left(\mathbf{H}^{T}(0) \mathbf{a}_{c}(n)\right)^{*}
$$

where $\mu_{1}$ is a real constant and $\boldsymbol{\Phi}_{c, 1}$ is given by

$$
\begin{aligned}
\boldsymbol{\Phi}_{c, 1} & =\sigma_{z, c}^{2} \sum_{\substack{m=-\infty \\
m \neq 0}}^{\infty}\left(\mathbf{H}^{T}(m) \mathbf{a}_{c}(n)\right)^{*}\left(\mathbf{H}^{T}(m) \mathbf{a}_{c}(n)\right)^{T} \\
& +\sum_{p=1}^{P} \sum_{m=-\infty}^{\infty} \sigma_{\tilde{z}_{p, c}}^{2} \tilde{\mathbf{h}}_{p, c}^{*}(m) \tilde{\mathbf{h}}_{p, c}^{T}(m)+\sigma_{n}^{2} \mathbf{I} .
\end{aligned}
$$

Similarly, for a fixed reception weight vector $\mathbf{b}_{c}(n)$, the optimal transmission weight vector is

$$
\left.\mathbf{a}_{c}(n)\right|_{\operatorname{SINR}_{\text {max }}, \mathbf{b}_{c}(n)}=\mu_{2} \boldsymbol{\Phi}_{c, 2}^{-1}\left(\mathbf{H}(0) \mathbf{b}_{c}(n)\right)^{*}
$$


where $\mu_{2}$ is a real constant and $\boldsymbol{\Phi}_{c, 2}$ is defined as

$$
\boldsymbol{\Phi}_{c, 2}=\sigma_{z_{c}}^{2} \sum_{\substack{m=-\infty \\ m \neq 0}}^{\infty}\left(\mathbf{H}(m) \mathbf{b}_{c}(n)\right)^{*}\left(\mathbf{H}(m) \mathbf{b}_{c}(n)\right)^{T}+\frac{\zeta}{P_{T}} \mathbf{I}
$$

with $\zeta=\mathbf{b}_{c}^{H}(n)\left[\sigma_{n}^{2} \mathbf{I}+\sum_{p=1}^{P} \sum_{m=-\infty}^{\infty} \sigma_{\tilde{z}_{p, c}}^{2} \tilde{\mathbf{h}}_{p, c}^{*}(m)\right.$ $\left.\tilde{\mathbf{h}}_{p, c}^{T}(m)\right] \mathbf{b}_{c}(n)$. Using (16) and (18), the optimal weight vectors for transmission and reception in frequency selective fading radio channels can be estimated iteratively. That is by guessing an initial value of $\mathbf{a}_{c}(n)$, we can find $\mathbf{b}_{c}(n)$ from (16). Using this $\mathbf{b}_{c}(n)$, we can then update our initial guess for $\mathbf{a}_{c}(n)$ using (18), and so on iteratively.

In order to have fast convergence, a good choice for an initial guess of $\mathbf{a}_{c}(n), \mathbf{a}_{c}^{(0)}(n)$ is important. A logical guess is

$$
\mathbf{a}_{c}^{(0)}(n)=\left[\begin{array}{llll}
1 & 1 & \cdots & 1
\end{array}\right]^{T}
$$

although better choices are available.

\section{COMPLEXITY REDUCTION}

As we can see, the calculation of the antenna weights for every subcarrier requires eigenvalue decomposition (EVD) as well as matrix inversion, and this could be enormous computation burden even at the base station, therefore, not making it feasible. However, in fact, the channel gain at a particular subcarrier frequency is not supposed to be totally different from its neighbouring frequencies, and hence, they must have correlation which depends on the coherence bandwidth of the channel, $B_{c}$.

Consider an OFDM system with transmission bandwidth of $5 \mathrm{MHz}$ and 1024 subcarriers. The subcarrier bandwidth is

$$
\Delta f=\frac{5 \times 10^{6}}{1024}=4.88 \mathrm{kHz} .
$$

Assume that the rms delay spread $\tau$ is $100 \mathrm{~ns}$, then the approximate coherence bandwidth is given by

$$
B_{c}=\frac{1}{50 \tau}=0.2 \mathrm{MHz} \approx 40 \Delta f .
$$

As a result, about 40 consecutive subcarriers are faded coherently, and this implies that it is possible to use the same set of antenna weights for a number of subcarriers instead of calculating smart antennas for every individual subcarrier. Using this approach, the computing complexity can be reduced significantly.

\section{SIMULATION RESULTS}

The proposed SBM/OFDM system introduced in Section II is investigated for a TDMA/TDD based wireless communication system by computer simulation. All channel state information is estimated using pilot tones. The configurations considered are for
OFDM systems with 1024 subcarriers and (a) 2 antenna elements at both the BS and MS, and (b) 2 antenna elements at BS and 3 antenna elements at MS. For each technique, average error probability $\left(P_{e}\right)$ is provided for various CCI (Signal-to-interference ratio (SIR)) and AWGN (SNR). The results are compared with a conventional adaptive antenna system where antennas are used at the BS only. Results for standard QPSK modulation without smart antennas are also listed for comparison. To refer to the different configurations, we use the notation $K+L$. For example, when we use SBM with 2 antennas at BS and 3 antennas at MS, we refer to it as $2+3 S B M$.

For each $P_{e}$ simulation, data packets consisting of 1,000 data symbols are transmitted with more than 10,000 independent channels [10]. For the transmitted signals, a raised cosine pulse shaping filter with rolloff factor of 0.22 is used. In the simulation, the channel of each link contains 10 random multipath components $(N=10$ in (3)) and one interferer.

\section{V-A. SBM/OFDM with Single Subcarrier}

The first simulation results are for the BER of a single subcarrier, and is equivalent to SBM under flat fading conditions. In Figure 3, results are provided in the absence of interference for QPSK, $1+2$ conventional, $1+3$ conventional and $2+2$ SBM. A close observation of this figure indicates that there is up to 100 times decrease in $P_{e}$ when SBM is employed instead of the conventional 2 elements smart antenna system at the BS only. This is an interesting result and reveals that significant advantages can be achieved by using smart antennas at both the BS and MS.

The configuration of SBM in Figure 3 involves 4 antenna elements ( 2 at the MS and 2 at the BS). To further investigate the potential of SBM compared with a conventional smart antenna system, we have listed in Figure 3 the simulation results of a conventional smart antenna system with 4 antennas (1 at the MS and 3 at the BS). By doing so, we have found that $\mathrm{SBM}$, for example, is about $2 \mathrm{~dB}$ better than the conventional antenna system. The advantages of SBM can be explained by realizing that one more diversity channel is utilized compared to the conventional system even if the total number of used antennas at the $\mathrm{BS}$ and MS are the same.

In Figure 4, similar results as those of Figure 3 are provided for a single subcarrier channel, but with CCI at $\mathrm{SIR}=15 \mathrm{~dB}$. These results demonstrate that SBM provides a 100 fold decrease in $P_{e}$ as compared to a smart antenna with 2 antennas at the BS only. The above results also demonstrate that more than a 10 fold reduction in $P_{e}$ is possible for the SBM compared with the conventional adaptive antenna system with 3 antenna elements.

V-B. SBM/OFDM in Frequency Selective Fading 
In Figure 5, we provide similar results as the ones given before over frequency selective fading channels which can be characterized by the normalized delay spread, $D$. The parameter $D$ is an important and convenient measure of the degree of frequency selective fading. In particular, in digital transmission over multipath channels, the BER is highly dependent on $D$. In fact, $D$ must be significantly smaller than unity in order to prevent excessive irreducible BER due to ISI in a conventional (single carrier) non-equalized modulation scheme [6].

In our simulations, for SBM/OFDM, we will exclusively let the total number of subcarriers $N_{c}$ be 1024, symbol period $T=0.2 \mathrm{~ms}$, and $\mathrm{rms}$ delay spread $\tau=100 \mathrm{~ns}$. On the other hand, for SBM, no frequency division multiplexing is done, the symbol period $T$ is then $195 \mathrm{~ns}$ in order to have the same throughput as that of OFDM and SBM/OFDM. Therefore, the rms delay spread normalized by the symbol period is 0.512 .

A close observation of Figure 5 reveals that more than a 10 fold reduction in $P_{e}$ is possible for the SBM system compared with the conventional OFDM. Likewise, for the SBM/OFDM system, it has more than a 1000 fold decrease in $P_{e}$ performance compared to the conventional OFDM without antenna diversity. It is noted that the results of SBM/OFDM is similar to that of SBM in flat fading channels.

For both SBM and SBM/OFDM, perfect channel information is required. For SBM/OFDM, the channel can be characterized by flat fading. Hence, the closed form SBM solution (Section III-A) can be used. However, for SBM in frequency selective channels, a more computationally extensive iterative process is required [5]. This will not only increase the complexity of the signal processing, but will also result in worse performance compared with that in flat fading.

\section{V-C. Complexity Reduction}

One may argue that the complexity of doing SBM for every subcarrier is also very high. As discussed previously, the weighted combining at the antennas can be done for groups of subcarriers instead of every individual subcarrier. As a result, the computational complexity can be reduced. Here, the coherence bandwidth of the channel, $B_{c}$, is roughly 40 adjacent subcarriers (See (22)). In Figure 6, simulation results are provided for $2+2 \mathrm{SBM} / \mathrm{OFDM}$, but calculating the antenna weights for a group of subcarriers. Group sizes of $1,40,80,120,160$, and 200 are considered.

Results show that there is less than $1 \mathrm{~dB} P_{e}$ performance degradation for group size of 40 compared with calculating the weights for every subcarrier (i.e. Group size equals 1). However, it is possible to further reduce the computing complexity with very little performance degradation. As a result, a group size of 80 seems the best choice where an 80 times reduction in computing complexity can be achieved with very little
$P_{e}$ performance degradation. In addition, group sizes of 120,160 , or 200 are provided and less than $10^{-4}$ $P_{e}$ can also be achieved. Therefore, it is possible to have even lower computing complexity to implement $\mathrm{SBM} / \mathrm{OFDM}$ when higher $P_{e}$ is tolerable.

\section{CONCLUSIONS}

Smart antennas have recently received a lot of attention in research and development of wireless personal communication systems. The investigation presented here has demonstrated the feasibility of utilizing smart antennas at the base and mobile stations in the up and downlinks. In particular, smart antenna can be applied to OFDM, namely SBM/OFDM. SBM/OFDM inherits the interference rejection capability of the smart antennas and ISI elimination of OFDM. Simulation results reveal that our system can reduce the average error probability by more than 1000 times for frequency selective fading channels. As such, this system guarentees excellent performance for high data rate wireless communications. In addition, users are able to be separated by time, frequency or space, therefore, enhancing the system capacity significantly.

The implementation of the system, however, requires that multiple antennas be incorporated into the MS and BS. Consequently, we suggest its suitability for wireless LAN applications in combination with recent advances in compact antenna design [11].

\section{REFERENCES}

[1] C. G. Günther, J. E. Padgett, and T. Hattori, "Overview of wireless personal communications," IEEE Commun. Mag., Vol. 33, No. 1, pp. 28-41, 1995.

[2] J. C-I Chuang, K. B. Letaief, K. F. Cheung, C. C. Ling, R. D. Murch, C. T. Nguyen and R. Yu, "Wireless Personal Communications in Hong Kong: A University Perspective," IEEE Personal Communications Magazine, Vol. 4, No. 2, pp. 30-43, Apr. 1997.

[3] J. H. Winters, "Signal acquisition and tracking with adaptive arrays in the digital mobile radio system IS-54 with flat fading," IEEE Trans. on Vehicular Technology, Vol. VT-42, No. 4, pp. 377-384, Nov. 1993.

[4] J. Fuhl, and A. F. Molisch, "Capacity enhancement and BER in a combined SDMA/TDMA system," Proceedings of IEEE 46th Vehicular Technology Conference, Vol. 3, pp. 1481-1485, New York, USA, 1996.

[5] K-K Wong, K. Ben Letaief, and R. D. Murch, "Investigating the performance of smart antenna systems at the mobile and base stations in the down and uplinks," Proceedings of 1998 IEEE Vehicular Technology Conference, Vol. 2, pp. 880-884, Ottawa, Canada, May 17-21 1998.

[6] J. C.-I. Chuang, 'The effects of time delay spread on portable radio communications channels with digital modulation," IEEE Journ. on Selected Areas in Communications, Vol. JSAC-5, No. 5, pp. 879-889, 1987.

[7] J. K. Cavers, "An analysis of pilot assisted modulation for Rayleigh fading channels," IEEE Trans. on Vehicular Technology, Vol. VT-40, No. 4 pp. 686-693, Nov. 1991.

[8] Jr. R. T. Comption, Adaptive Antennas Concepts and Performance, Prentice-Hall, New Jersey, 1988.

[9] G. Strang, Linear Algebra and its Applications, Harcourt Brace Jovanovich, San Diego, 1988.

[10] J. C-L Ng, K. B. Letaief, and R. D. Murch, "Trade-offs between diversity combining and equalization for wireless LANs," Proceedings of 1997 IEEE Vehicular Technology 


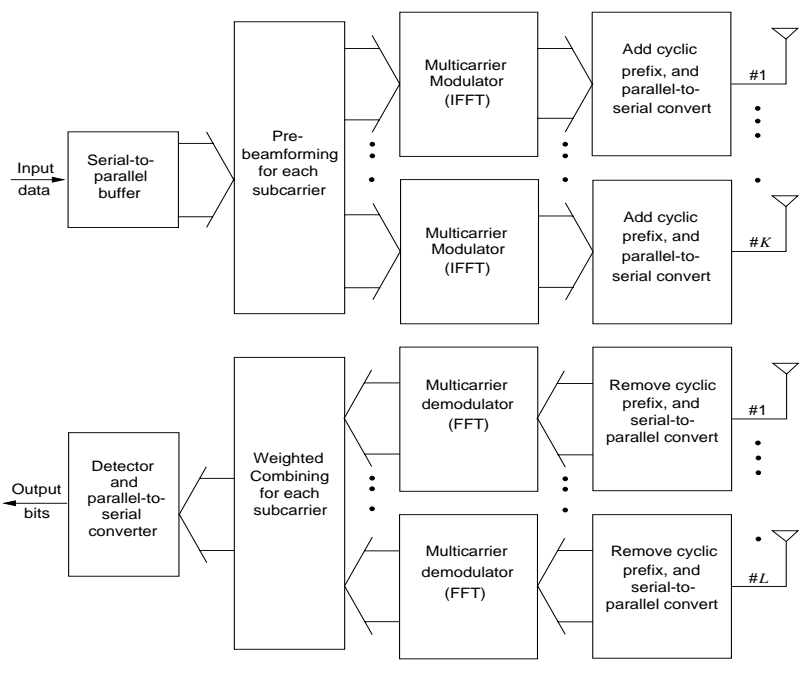

Fig. 1. System configuration of SBM/OFDM.

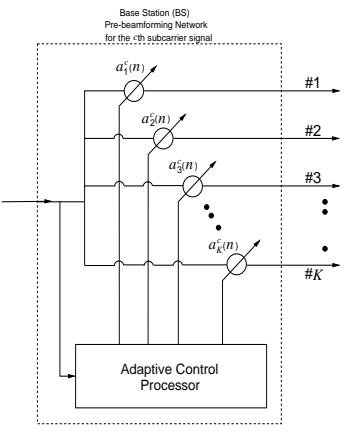

(a)

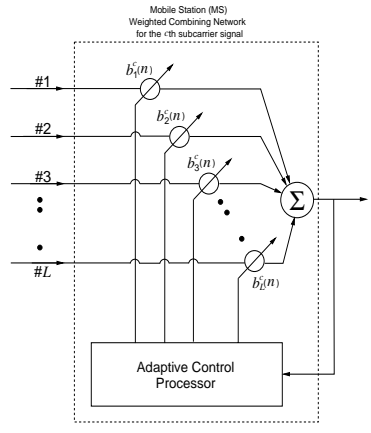

(b)
Fig. 2. System configuration of (a) pre-beamforming, and (b) weighted combining networks at the BS and MS.

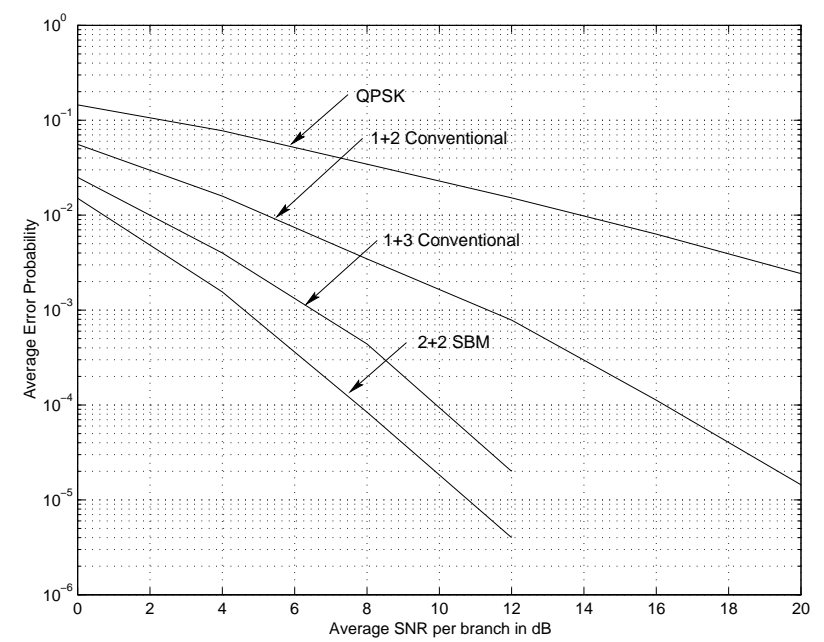

Fig. 3. Average error probability performance of SBM under conditions of no interference and flat fading.

Conference, pp. 875-879, Pheonix, Arizona, USA, May 4-7 1997.

[11] C. R. Rowell, and R. D. Murch, "A Compact PIFA suitable for dual frequency $900 / 1800 \mathrm{MHz}$ operation," Accepted in IEEE Transactions on Antennas and Propagation.

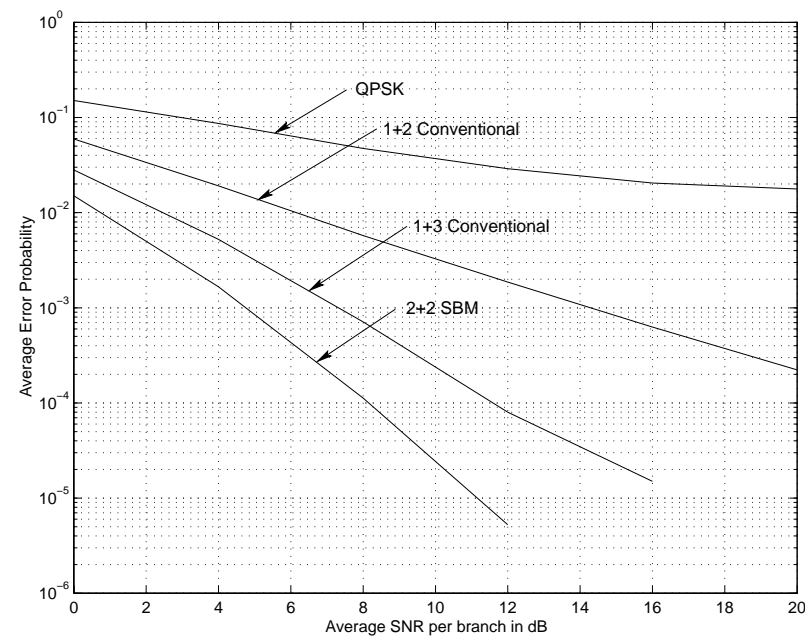

Fig. 4. Average error probability performance of SBM in flat fading conditions with a single dominant CCI at $\mathrm{SIR}=15 \mathrm{~dB}$.

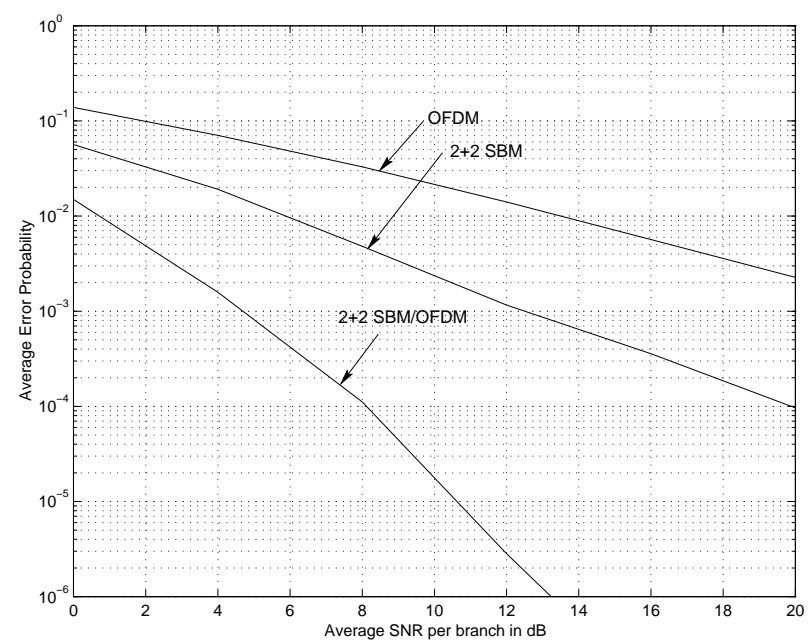

Fig. 5. Average error probability performance of SBM/OFDM under conditions of no interference and multipath fading channels.

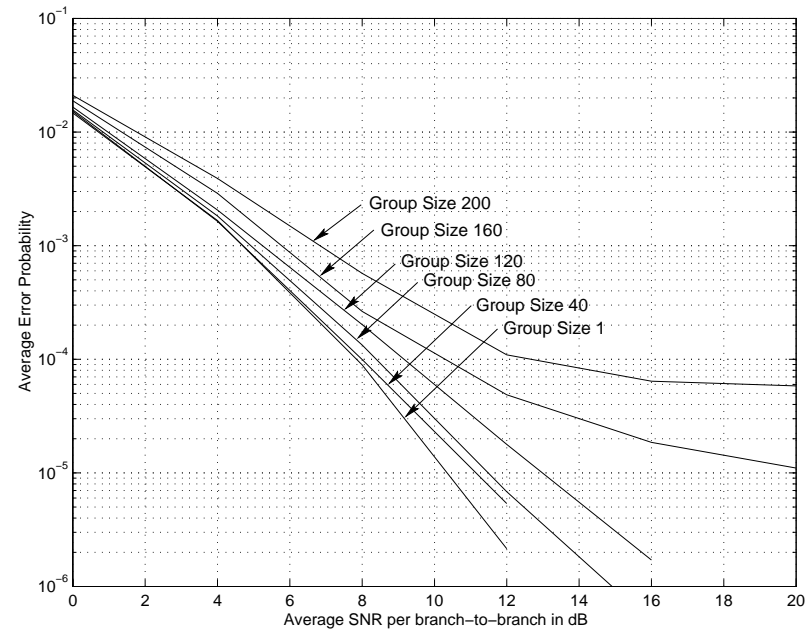

Fig. 6. Average error probability performance of SBM/OFDM in frequency selective fading channels. 